\title{
UNIQUE COLLECTION OF ORIOLE NESTS
}

JIM A. WEDGWOOD, 610 Leslie Avenue, Saskatoon, Saskatchewan, S7H 22 Photographs by THELMA and JIM PEPPER, 1015 Temperance Street, Saskatoon, Saskatchewan. S7N ON5

In the late 1940s, the Opseth family, Hagen district farmers, started collecting oriole nests, and Otto Opseth now continues the practice started by his sister. Most of the nests are from an aspen grove surrounding the farmhouse.

Settlers in northeastern Saskatchewan often first cleared a small opening in the bush for a farmyard. Then, when breaking the land, they left the trees surrounding the yard standing, thus providing a ready-made shelterbelt. Many of these belts remain and typically house a pair ororioles, a couple of crested flycatchers and one or more pairs of hummingbirds, among other species. With two-thirds of a total yard area of 12 acres being treed, the Opseth place follows this pattern.

Orioles have nested every year. When he was younger, Otto used to climb the tree at the end of the season to get the nest. Later he tried shooting off the branch, a largely unsuccessful venture. Now he cuts down the tree - "they need thinning anyway" - hoping to beat the magpies, who tear open the nests looking for parasites. In one nest fleas lined the bottom to a thickness of $1 / 8$ in.

Otto displayed the nests when Thelma and Jim Pepper led a group of fieldtrippers to his farm in 1988. The Peppers and I went back in October 1989, they to photograph the nests, I to study them. The nests selected were about a third of the collection and are representativ nestbuilding techniques and factors fluencing them.

The customary placement of the r was high in a tall tree, but in the cas Nest I the choice of a hawthorn b meant a much lower nest. Nest VI ( photos) was also in a shrub, at $10 \mathrm{ft}$. only other low nest in the group. On bird has selected a tree, what trigge to choose a particular nest site and wh precisely to initiate suspension were unclear.

Nest l's suspension was the simpl a mere loop of only a few strands. Sd of the other nests had most intric suspensions - Nest Illa, for exam The reason for the difference may that there were no twigs or brand near the initial point of attachmen Nest I. On the other hand, there wer least four branches close to the to Nest IIla, and the bird anchored her to each one. Similarly, alongside $\mathrm{Ne}$ were four branches, one below the of and again the bird made attachmen each as it worked its way down, or uff the case may be.

A considerable variety of mate was used in the nests. Nest I, thoug, a non-indigenous material - horsel may be the pure form of oriole having minimum volume, least mat and, as a result, least energy expe ture in construction. This nest was 


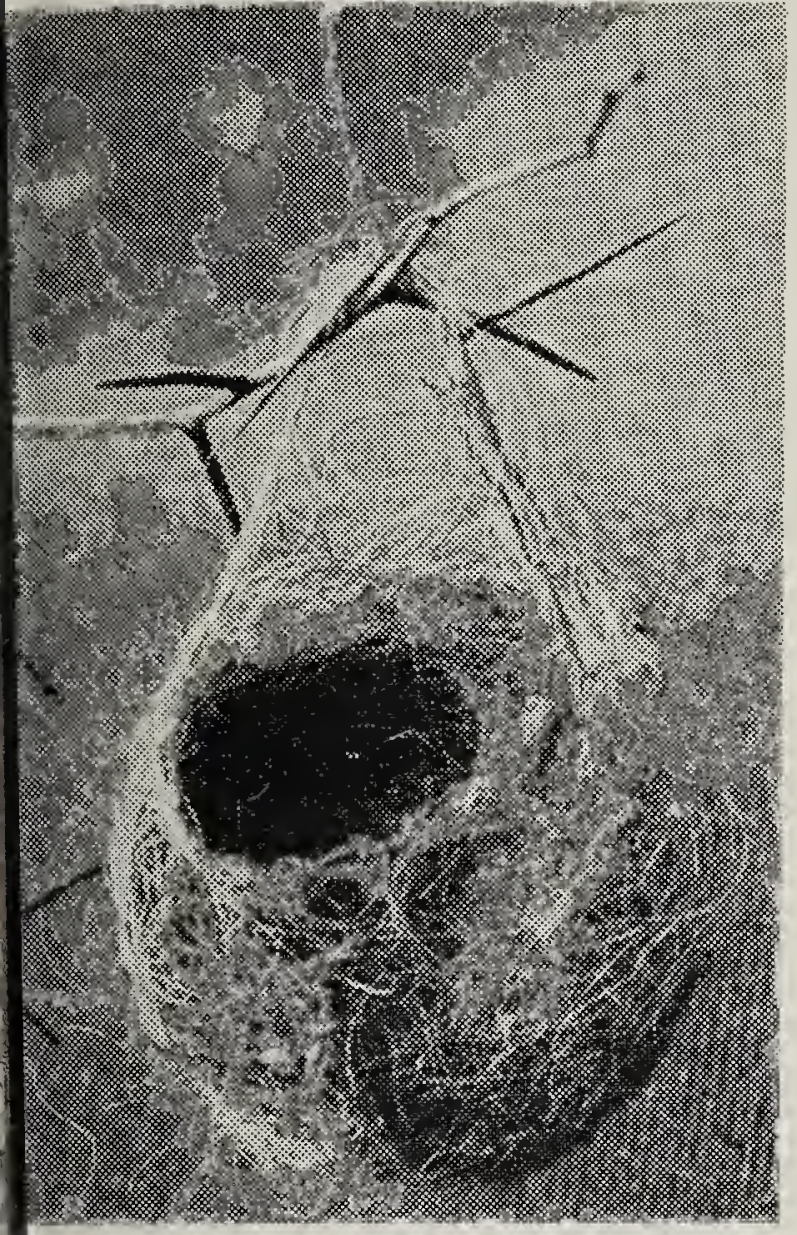

11: Oriole nest, $100 \%$ horsehair

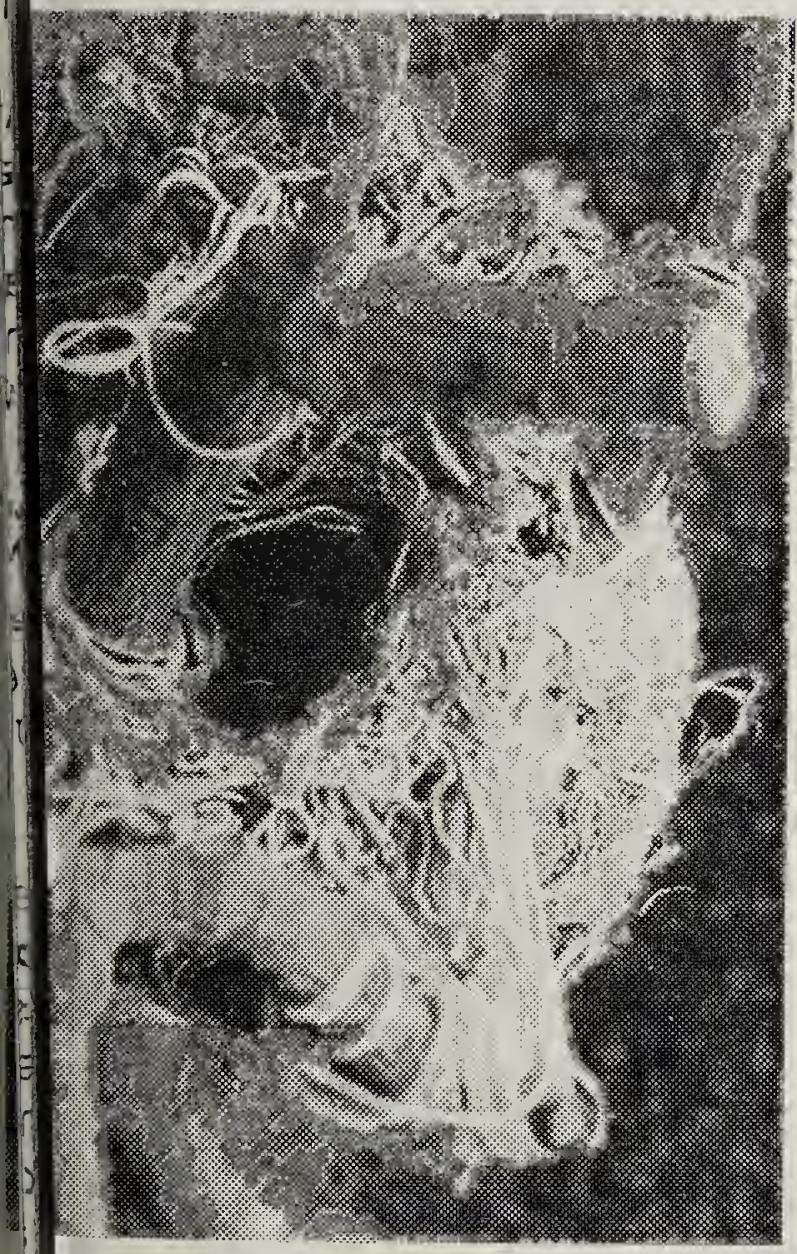

IIla : Oriole nest, $75 \%$ string , 25\% bark

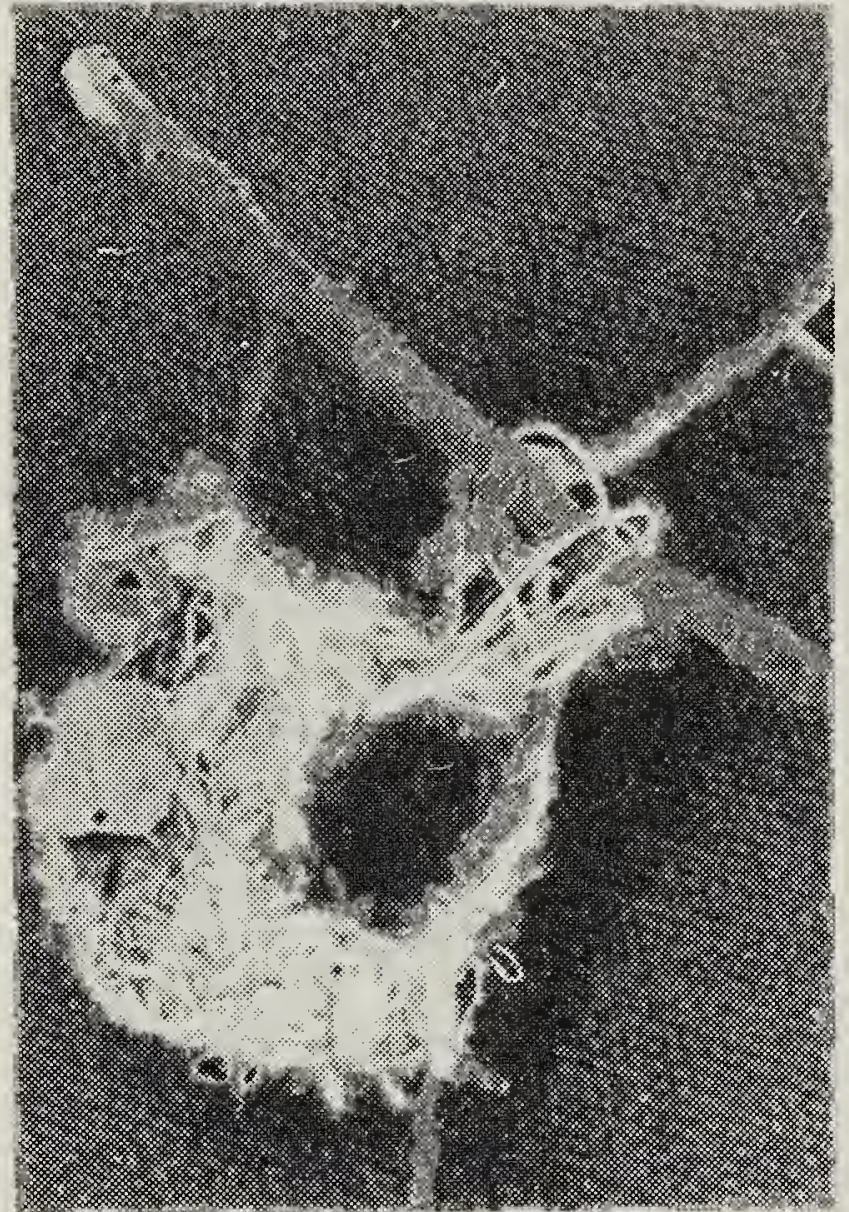

Nest II: Oriole nest, 50\% horsehair, 50\% string

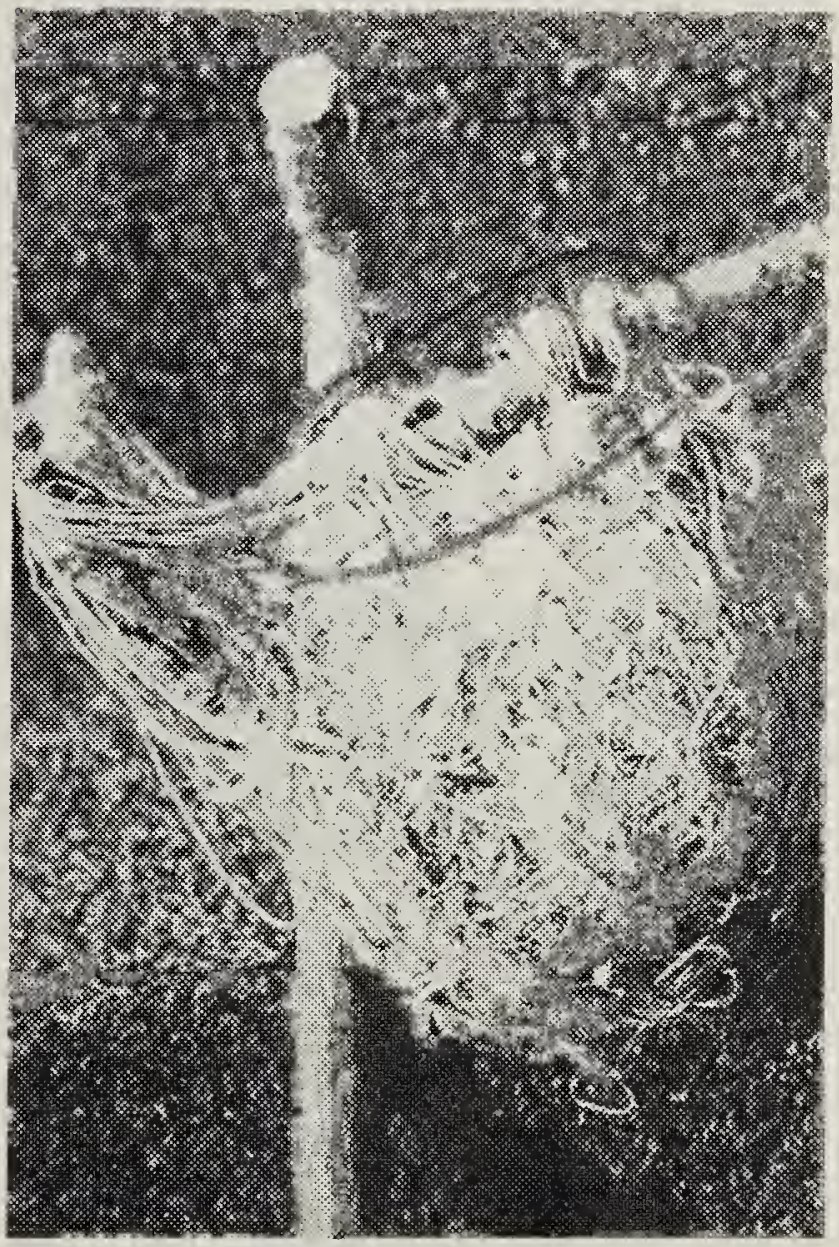

Nest II/b: Oriole nest, 75\% string, 25\% bark fibre 




Nest IV : Oriole nest, $100 \%$ bark fibre

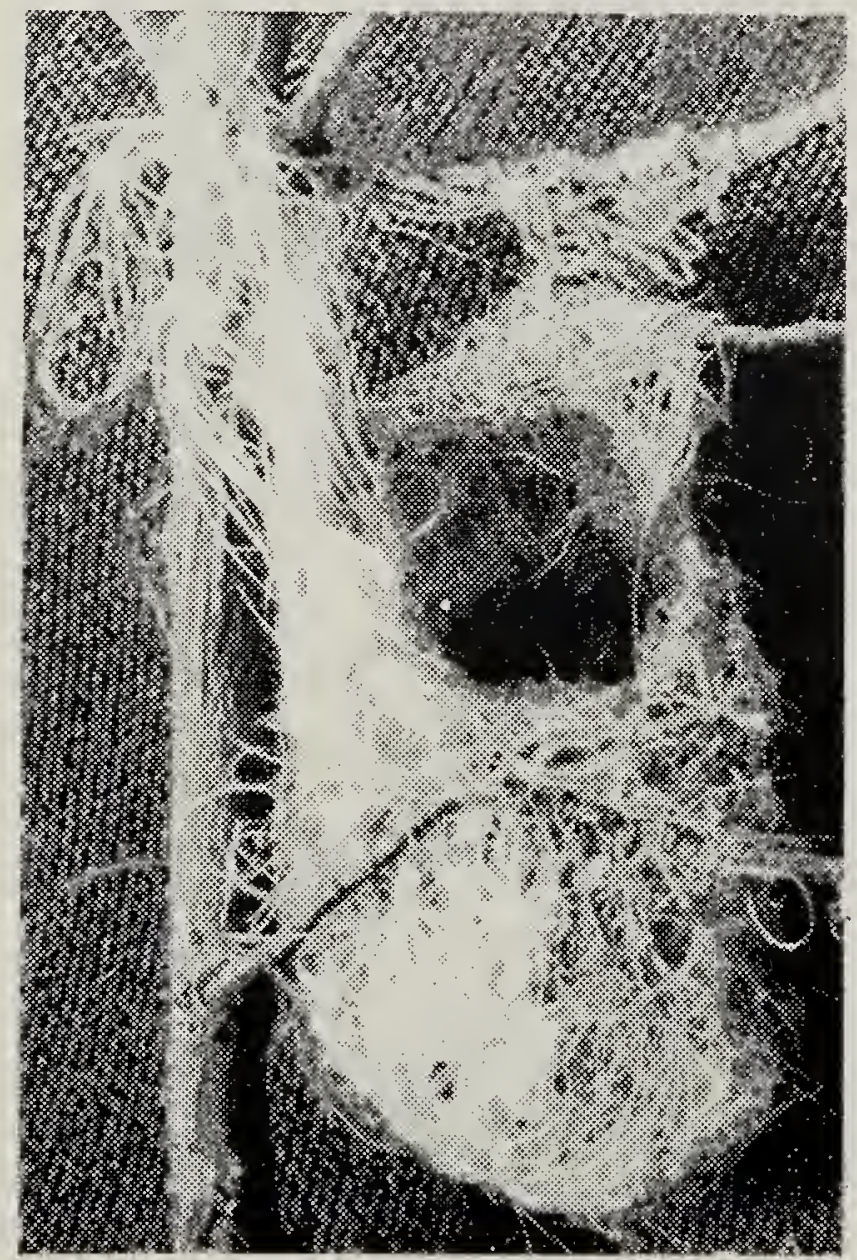

Nest VI: Oriole nest, $100 \%$ synthetic twine

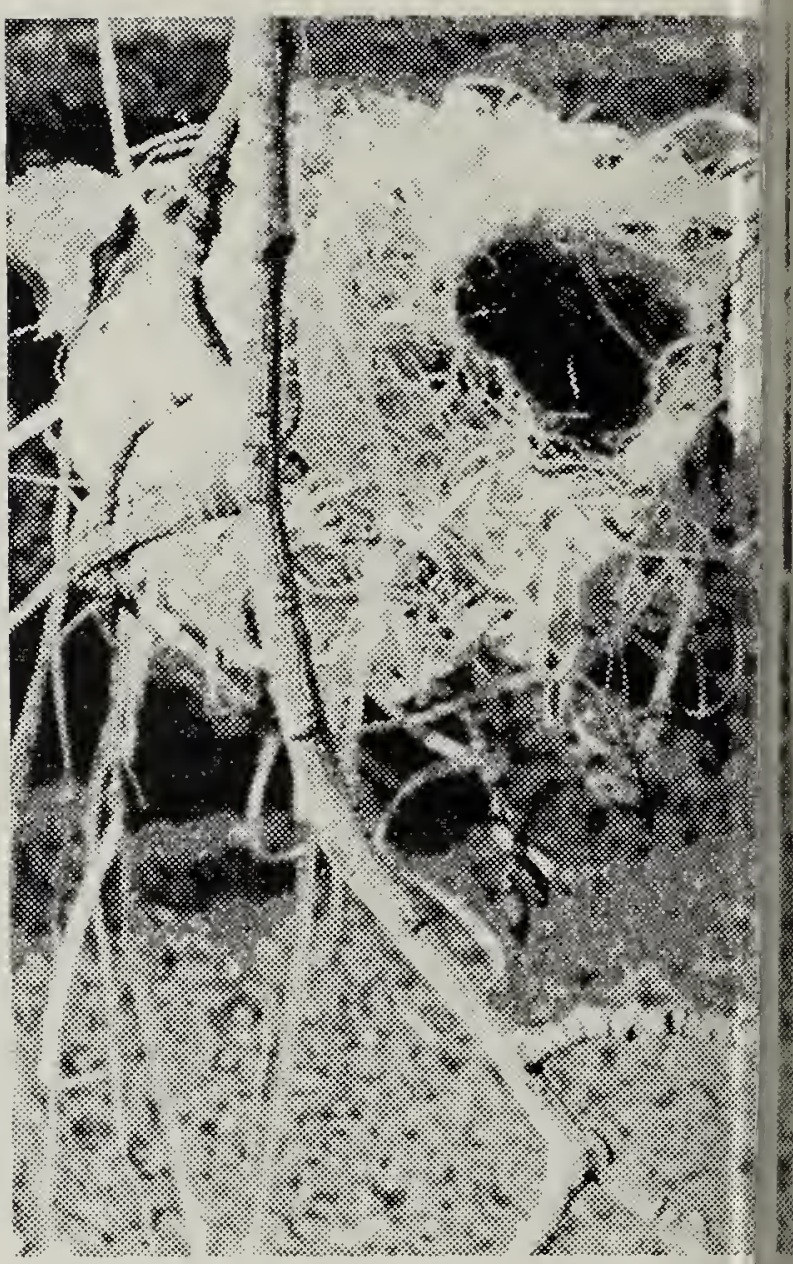

Nest V : Oriole nest, $100 \%$ synthetic y

$4 \mathrm{in.}$ by $41 / 4 \mathrm{in}$. by $3 \mathrm{in}$. high to the of the opening.

The oldest, smallest and simple in the collection, Nest I was notable in 1 ;e respects: ideal suspension, efficient $m$ and optimum use of materials. En ly of horsehair, it reflects a way of far ng now gone.

The Opseths had hung out ort lengths of string for nest material an he birds readily took them (Nests II an II). Accompanied by the male, the fe lle would fly down, pick up a length, fly ck to the nest and weave it in, still acco ranied by the male, who, typical c he species, was in constant attendanc ut did no work.

Coarseness of string made no $($ erence, but colour did. The birds took ite, blue or purple, but not red - not $2 e$, but repeatedly and over several y irs. Their instinct may have been to oid 
trong colours or colours markedly conasting with surroundings. To human yes, however, that explanation does ot fit with what we see in Nest VI. That est, made of synthetic twine, was still $n$ eye-catching shiny white when the est was taken down.

Nests $V$ and $V I$ were of the Plastic ge, one made of flat nylon cord from eed grain bags (robins used it too), the her of a teased synthetic twine.

The only truly natural nest in the sected group was Nest IV, which was ade solely of bark fibres. The other five sed one or more non-indigenous mateals. For some reason, the lower part of is nest was $1 / 4$ in. thick, much thicker an in the other nests. One can see rough the bottoms of most of them. here was a decided thermal advantage a thick layer of bark fibre. To begin th, it has a higher insulating value rand for strand than either plastic or prsehair.

The orioles were not constrained to e one material throughout. Nest II is $\%$ string and $50 \%$ horsehair. Nest III $75 \%$ string, $25 \%$ bark fibres and some rsehair.

The weaving appeared most random, pking more like felting on some nests. wever, on Illb (the side view of Nest a) interlacing of lateral and suspending ands may be seen immediately below cross twig (this section is circled on photograph).
The typical oval opening was 2 in. by $31 / 2$ in. Compared to this, the $11 / 4$ in. diameter hole in Nest Illa was small. The year of that nest, 1979, saw a tent caterpillar infestation in the district. The trees were defoliated, exposing the nest, and though the birds stayed on, they acted shy (Northern Orioles are among the few species that will eat hairy larvae such as tent caterpillars ${ }^{2}$ ). One view is that the presence of the caterpillars caused the bird to make a smaller opening. Another opinion is that in this particular case the inherent urge to secure the nest to every nearby twig inevitably led to a small round opening.

A female oriole is $7 \mathrm{in}$. long from tail tip to bill tip, twice the length of her nest. ${ }^{1}$ This means that while incubating she has to adopt a U-shape, head and tail pointed up and almost touching.

A collection of nests such as the Opseths' provides insights into variations in building techniques and into results of habitat changes, including impacts of human activities. We are grateful to Mrs. Helen Opseth and Otto Opseth for making the nests available and providing information about them. We greatly appreciated their kind assistance during our visit.

'GODFREY, W. E. 1986. The birds of Canada. National Mus. of Canada, Ottawa. 595 $\mathrm{pp}$.

2SALT, W. R. and J. R. SALT. 1976. The birds of Alberta. Hurtig, Edmonton. 498 pp. 